\title{
Length of Stay, Hospital Costs and Mortality Associated With Comorbidity According to the Charlson Comorbidity Index in Immobile Patients After Ischemic Stroke in China: A National Study
}

\author{
Hongpeng Liu' ${ }^{\mathbb{1}}$, Baoyun Song ${ }^{2}$, Jingfen Jin ${ }^{3}$, Yilan Liu ${ }^{4}$, Xianxiu Wen ${ }^{5}$, Shouzhen Cheng ${ }^{6}$, Stephen \\ Nicholas $^{7,8,9,10}$, Elizabeth Maitland ${ }^{11}$, Xinjuan $\mathrm{Wu}^{1^{*}}{ }^{\mathbb{D}}$, Dawei $\mathrm{Zhu}^{12^{*} \mathbb{D}}$
}

\begin{abstract}
Background: In this study, we examined the length of stay (LoS)-predictive comorbidities, hospital costs-predictive comorbidities, and mortality-predictive comorbidities in immobile ischemic stroke (IS) patients; second, we used the Charlson Comorbidity Index (CCI) to assess the association between comorbidity and the LoS and hospitalization costs of stroke; third, we assessed the magnitude of excess IS mortality related to comorbidities.

Methods: Between November 2015 and July 2017, 5114 patients hospitalized for IS in 25 general hospitals from six provinces in eastern, western, and central China were evaluated. LoS was the period from the date of admission to the date of discharge or date of death. Costs were collected from the hospital information system (HIS) after the enrolled patients were discharged or died in hospital. The HIS belongs to the hospital's financial system, which records all the expenses of the patient during the hospital stay. Cause of death was recorded in the HIS for 90 days after admission regardless of whether death occurred before or after discharge. Using the CCI, a comorbidity index was categorized as zero, one, two, and three or more CCI diseases. A generalized linear model with a gamma distribution and a log link was used to assess the association of LoS and hospital costs with the comorbidity index. Kaplan-Meier survival curves was used to examine overall survival rates.

Results: We found that $55.2 \%$ of IS patients had a comorbidity. Prevalence of peripheral vascular disease (21.7\%) and diabetes without end-organ damage $(18.8 \%)$ were the major comorbidities. A high CCI=3+ score was an effective predictor of a high risk of longer LoS and death compared with a low CCI score; and CCI $=2$ score and CCI=3+ score were efficient predictors of a high risk of elevated hospital costs. Specifically, the most notable LoS-specific comorbidities, and cost-specific comorbidities was dementia, while the most notable mortality-specific comorbidities was moderate or severe renal disease.

Conclusion: CCI has significant predictive value for clinical outcomes in IS. Due to population aging, the CCI should be used to identify, monitor and manage chronic comorbidities among immobile IS populations.

Keywords: Stroke, Comorbidity, Mortality, Costs, Length of Stay

Copyright: (c) 2022 The Author(s); Published by Kerman University of Medical Sciences. This is an open-access article distributed under the terms of the Creative Commons Attribution License (https://creativecommons.org/licenses/ by/4.0), which permits unrestricted use, distribution, and reproduction in any medium, provided the original work is properly cited.

Citation: Liu H, Song B, Jin J, et al. Length of stay, hospital costs and mortality associated with comorbidity according to the Charlson comorbidity index in immobile patients after ischemic stroke in China: a national study. Int J Health Policy Manag. 2022;11(9):1780-1787. doi:10.34172/ijhpm.2021.79
\end{abstract}

Article History:

Received: 11 December 2020 Accepted: 3 July 2021 ePublished: 7 August 2021

\section{Background}

Stroke is the second most common cause of death and disability worldwide. $^{1-3}$ Globally, there were 80.1 million prevalent cases of stroke, with $84.4 \%$ ischemic stroke (IS). ${ }^{4}$ In 2016, 5.5 million died of stroke, and IS accounted for $49.1 \%$ of all stroke deaths. ${ }^{4}$ Stroke has been the leading cause of death in China in recent years, ${ }^{5}$ with 1.8 million annual deaths, accounting for roughly $30 \%$ of worldwide stroke mortality. ${ }^{5-7}$ Stroke also imposes a significant economic burden on society, families and individuals. ${ }^{8}$ In 2015, the annual cost for stroke care was estimated to RMB37.5 billion, ${ }^{9}$ and 2017 hospitalization cost per IS patient was RMB10 131, while China's per capita disposable income was only RMB25974. ${ }^{10}$ With increasing life expectancy and population aging, the stroke burden will pose an even more serious healthcare and economic challenge to China. ${ }^{11}$

Stroke sufferers commonly have comorbidities, such as hypertension, cardiovascular disease, diabetes, or other ailments, including bleeding, medical complications, or stroke recurrence, ${ }^{12-14}$ that directly or indirectly present barriers to optimal stroke recovery. ${ }^{15,16}$ China has 7.5 million stroke survivors who suffered from comorbidities. ${ }^{17}$ These stroke survivors usually spend a significant period immobilized in bed, ${ }^{2,18,19}$ leading to personal functional limitations, loss of muscle mass, and medical complications. ${ }^{20,21}$ Comorbidity and immobility have a substantial effect on the final outcome of stroke patients, and often impede recovery, impacting directly or indirectly length of stay (LoS) in hospital, hospital 


\section{Key Messages}

Implications for policy makers

- The Charlson Comorbidity Index (CCI) has significant predictive value for clinical outcomes in immobile patients with ischemic stroke (IS).

- Health insurance plans should use the CCI to assess and identifies the complexity of stroke-related diseases and the current disease status (such as hypertension, dementia, and diabetes) of the insured member.

- $\quad$ For clinical staff, CCI should be used to assess IS patients most likely to experience the worse clinical outcomes and assess, monitor and treat comorbidities during hospitalization.

- Due to population aging, the CCI should be used to identify, monitor and manage chronic comorbidities among the immobile IS population. We also recommend establishing a geriatric care system, where the CCI can be optimized in patient treatment.

Implications for the public

Stroke sufferers commonly have comorbidities, such as hypertension or diabetes, that directly or indirectly present barriers to optimal stroke recovery. The results indicate that comorbidities were central drivers of length of stay (LoS), hospital cost, and mortality in Chinese immobile ischemic stroke (IS) patients. Assessing the association between comorbidity and the LoS and hospitalization costs of stroke, and understanding the magnitude of excess IS mortality related to comorbidities, promises both to save the lives of stroke sufferers through integrating medical interventions according to their comorbidities and to better manage hospital costs of IS stroke patients with comorbidities.

costs, and death. ${ }^{15,16}$

The impact of comorbidity on survival after hemorrhagic stroke among dialysis patients has been studied in Taiwan province. ${ }^{22}$ Yang et al examined the mortality-specific comorbidity among inpatients with IS in Sichuan province from 2012 to $2017 .{ }^{23}$ However, in China, there has been no nation-wide research on the LoS-specific comorbidities, hospital costs-specific comorbidities, and mortality-specific comorbidities of immobile patients with IS. Assessing the association between comorbidity and the LoS and hospitalization costs of stroke, and understanding the magnitude of excess IS mortality related to comorbidities, promises both to save the lives of stroke sufferers through integrating medical interventions according to their comorbidities and to better manage hospital costs of IS stroke patients with comorbidities. ${ }^{24}$ This study examines the LoS-predictive comorbidities, hospital costs-predictive comorbidities, and mortality-predictive comorbidities in immobile IS patients; second, we used the Charlson Comorbidity Index (CCI) to assess the association between comorbidity and the LoS and hospitalization costs of stroke; third, we assessed the magnitude of excess IS mortality related to comorbidities. We use a unique, large scale hospital-based multicenter dataset with an exhaustive discharge diagnosis to measure the stroke burden nationally of immobility IS patients with comorbidities.

\section{Materials and Methods}

\section{Study Design and Population}

Supported by National Health and Family Planning Commission's agenda to improve the outcomes among immobile patients with stroke, the target population is all immobile stroke inpatients in 25 general hospitals in China. In order to ensure the representativeness of the study sample, between November 2015 and July 2017, we collected stroke patient data in 25 general hospitals in eastern China (Guangdong province, Zhejiang province, and Beijing municipal city), western China (Sichuan province), and central China (Henan province and Hubei province), ${ }^{19}$ comprising six tertiary hospitals, 11 secondary hospitals, and eight community hospitals. Study subjects met the following inclusion criteria: ( $i$ ) aged 18 years or older; (ii) immobile, where the patient's basic physiological needs were carried out in bed, except for active or passive bedside sitting/standing/ wheelchair use for examination; (iii) ability to understand the study's aims and to sign the consent form; (iv) diagnosed with IS as the major illness in their medical records. IS was defined according to the World Health Organization (WHO) definition as focal neurological deficit lasting for 24 hours with no apparent other than vascular cause, ${ }^{25}$ which was coded I63. $x$ in the International Classification of Disease, 10th revision (ICD-10) based on pathological subtypes. ${ }^{2,26,27}$ All eligible inpatients of the selected hospitals were continuously enrolled. A total of 5114 participants enrolled in the study, with follow-ups continuing 90 days after enrollment unless they died in hospital or relinquished medical treatment. Survival time was measured by the duration from the date of hospital admission to the date of death and LoS was defined as the period from the date of admission to the date of hospital discharge or date of death, which is an important performance indicator of hospital management. ${ }^{28-30}$ Hospital costs were derived from the hospital information system (HIS) in each hospital after the enrolled patients died or were discharged from hospital. The HIS belongs to the hospital's financial system, which records all the expenses of the patient during the hospital stay. The listed cause of death of the IS patient was recorded in the HIS for 90 days after the date of admission regardless of whether death occurred before or after discharge. Dates of death were collected from standardized HIS case report forms.

\section{Definition of CCI and Covariates}

Comorbidity was assessed using the CCI, which identifies multiple comorbidities. Adjusted specifically for stroke evaluation, ${ }^{31-33}$ the CCI accounts for multiple comorbidities by creating a sum score, weighted according to the presence of 19 comorbid conditions..$^{16,26,34}$ We used the actual weighted score derived from the CCI. Different comorbid conditions had different weights in CCI. For example, Table 1 shows that myocardial infarction is weighted 1 ; diabetes with end-organ 
Table 1. Sociodemographic and Clinical Characteristics of the Patients $(\mathrm{N}=5114)$

\begin{tabular}{|c|c|}
\hline Variable & Number (\%) \\
\hline \multicolumn{2}{|l|}{ Gender } \\
\hline Male & $3028(59.2)$ \\
\hline Female & $2086(40.8)$ \\
\hline \multicolumn{2}{|l|}{ Age } \\
\hline $0-59$ & $1278(25.0)$ \\
\hline $60-69$ & $1348(26.4)$ \\
\hline $70-79$ & $1340(26.2)$ \\
\hline $80+$ & $1148(22.4)$ \\
\hline \multicolumn{2}{|l|}{ Education } \\
\hline Illiteracy & $1037(20.3)$ \\
\hline Primary school & $1781(34.8)$ \\
\hline Junior high school & $1226(24.0)$ \\
\hline High school and above & 1070 (20.9) \\
\hline \multicolumn{2}{|l|}{ Insurance } \\
\hline No insurance ${ }^{a}$ & 815 (15.9) \\
\hline NCMS & $2070(40.5)$ \\
\hline URBMI & $981(19.2)$ \\
\hline UEBMI & $1248(24.4)$ \\
\hline \multicolumn{2}{|l|}{$\mathrm{CCl}$ score } \\
\hline 0 & $2291(44.8)$ \\
\hline 1 & $1600(31.3)$ \\
\hline 2 & $704(13.8)$ \\
\hline $3+$ & $519(10.1)$ \\
\hline \multicolumn{2}{|l|}{ Individual CCl diseases (Ref = Without comorbidity) } \\
\hline Myocardial infarction (weight $=1$ ) & $63(1.2)$ \\
\hline Congestive heart failure (weight $=1$ ) & $576(11.3)$ \\
\hline Peripheral vascular disease (weight $=1$ ) & $1108(21.7)$ \\
\hline Dementia (weight = 1) & $153(3.0)$ \\
\hline Chronic pulmonary disease (weight $=1$ ) & $468(9.2)$ \\
\hline Connective tissue disease (weight $=1$ ) & $61(1.2)$ \\
\hline Ulcer disease (weight = 1) & $49(1.0)$ \\
\hline Mild liver disease $($ weight $=1$ ) & $304(5.9)$ \\
\hline Diabetes without end-organ damage $($ weight $=1)$ & $960(18.8)$ \\
\hline Hemiplegia (weight = 2) & $15(0.3)$ \\
\hline Moderate or severe renal disease (weight $=2$ ) & 199 (3.9) \\
\hline Diabetes with end-organ damage (weight $=2$ ) & $106(2.1)$ \\
\hline Tumor without metastasis (weight $=2$ ) & $150(2.9)$ \\
\hline Leukemia (weight = 2) & $2(0.0)$ \\
\hline Lymphoma (weight = 2) & $6(0.1)$ \\
\hline Moderate or severe liver disease $($ weight $=3$ ) & $53(1.0)$ \\
\hline Metastatic solid tumor (weight $=6$ ) & $41(0.8)$ \\
\hline AIDS (weight = 6) & $3(0.1)$ \\
\hline Total & $5114(100)$ \\
\hline
\end{tabular}

Abbreviations: CCl, Charlson Comorbidity Index; NCMS, New Cooperative Medical System (covered rural residents); URBMI, Urban Resident Basic Medical Insurance (covered urban residents without a stable job); UEBMI, Urban Employee Basic Medical Insurance (covered employed workers); AIDS, acquired immune deficiency syndrome.

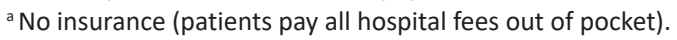

damage is weighted 2; and moderate or severe liver disease is weighted $3 .{ }^{23,26,34,35} \mathrm{CCI}$ was designed to enable researchers to control for the prognostic impact of other chronic diseases on the outcomes of patients with a specific disease, and it is commonly used in outcome and mortality studies. ${ }^{15,34,36}$ The CCI data in our study were derived from the discharge ICD-10 codes and patient histories obtained from the HIS standardized case report forms. Following previous studies, the total CCI score for each patient was categorized into four levels of comorbidity, 0 (none), 1 (moderate), 2 (severe), and $3+$ (very severe).

We identified all 19 comorbidities included in the CCI, ${ }^{15,36,37}$ except for cerebrovascular disease. Control variables included gender (male or female), age (0-59, 60-69, 70-79, 80 years or older), education level (illiteracy, primary school, junior high school, high school and above), and type of insurance. ${ }^{38}$ The type of insurance comprised no insurance, New Cooperative Medical System (NCMS) for rural residents, ${ }^{39}$ Urban Resident Basic Medical Insurance (URBMI) for urban unemployed, elderly, children and students, ${ }^{40}$ and Urban Employee Basic Medical Insurance (UEBMI) for urban employed and retired. ${ }^{41}$ NCMS, UEBMI and URBMI were China's three basic social health insurance schemes, but differed in reimbursement ratios, coverage and contributions. ${ }^{38}$ Previous research indicated that medical insurance was associated with the cost and adverse clinical outcomes of stroke, ${ }^{10,42-44}$ therefore, we included the type of insurance in the analysis.

\section{Statistical Analyses}

We employed descriptive statistics to summarize patients' characteristics. In the univariate analysis, LoS and hospital costs were compared between different subgroups using the rank-sum test, and death proportions were compared between different subgroup using the $\chi^{2}$ test. Generalized linear model with a gamma distribution and a log link was used to assess the association of LoS and hospital costs with the CCI score. The results were reported as percentage changes $\left(=\exp ^{\wedge \text { coefficient }-1)}\right.$ and $95 \%$ confidence intervals (CIs) in LoS and hospital costs for IS, in association with the presence of comorbidities. Kaplan-Meier survival curves was used to examine overall survival. Using multivariable Cox regression models, adjusted for gender, age, education level, and type of insurance, the relative risk of death of all mortality causes was expressed as hazard ratios (HRs) with 95\% CIs. In order to further analyze the specific types of comorbidity, we identified the nine most prevalent types of comorbidities as binary variables: peripheral vascular disease, diabetes without end-organ damage, congestive heart failure, chronic pulmonary disease, mild liver disease, moderate or severe renal disease, dementia, tumor without metastasis, and diabetes with end-organ damage. A $P$ value of less than .05 was considered statistically significant. Statistical analysis was performed with Stata version 14 for Windows (Stata Corp, College Station, TX, USA).

Results

Demographic Information of Stroke Patients

Descriptive statistics are shown in Table 1 and Table 2. 
Among the 5114 participants, 3028 (59.2\%) were male; there is no statistical difference between males and females in terms of the LoS (18 days - standard deviation [SD] \pm 15.4 ) versus 17 days $(\mathrm{SD} \pm 13.2 ; P=.069)$ and death $(7.4 \%$ versus. $6.5 \%$; $P=.211$ ), while there is a significance difference between the average hospital costs of males and females (RMB40 047 [SD \pm RMB57 705.5] versus RMB35339 [SD \pm RMB48152.9]; $P<.001)$. Three quarters of the participants were older than 60 years, and $22.4 \%$ older than 80 years. Participants younger than 59 years had higher average hospital costs than the elderly, while increasing age increased the risk of mortality. More than one third of participants had a primary school background and $20.9 \%$ had a high school and above education level. With regard to insurance type, 2070 (40.5\%) participants were covered by NCMS, $24.4 \%$ by UEBMI and $19.2 \%$ by URBMI, and $15.9 \%$ had no insurance. The proportion of participants with no comorbidities was $44.8 \%$, with $31.3 \%$ with one, $13.8 \%$ with two and $10.1 \%$ with three or more comorbidities. The most frequent comorbid conditions in Table 1 were peripheral vascular disease $(21.7 \%)$, diabetes without end-organ damage (18.8\%), congestive heart failure (11.3\%), chronic pulmonary disease $(9.2 \%)$, and mild liver disease (5.9\%). There is a significance difference between the different CCI scores in terms of the LoS, costs, and death $(P<.001)$. The data for hospitalizations, analyzed according to CCI scores, are presented in Table 2.
Length of Stay

Table 2 also displays the LoS among the study participants, with average $\mathrm{LoS}$ in the $\mathrm{CCI}=0$ group 17 days $(\mathrm{SD} \pm 13.2)$; $\mathrm{CCI}=1$ group 16 days ( $\mathrm{SD} \pm 13.8), \mathrm{CCI}=2$ group 18 days $(\mathrm{SD} \pm 13.2)$ and $\mathrm{CCI}=3+$ group 21 days $(\mathrm{SD} \pm 22.0)$. Figure 1 depicts the percentage change and $95 \%$ confidence interval (CI) in LoS associated with comorbidities. After adjusting for potential confounders (Table S1, see Supplementary file 1), compared with $\mathrm{CCI}=0$ score, there was no significance differences in $\mathrm{CCI}=1(0.9 \%, 95 \% \mathrm{CI}-0.039$ to $0.061 ; P=.712)$ and $\mathrm{CCI}=2$ (4.2\%, 95\% CI -0.025 to $0.113 ; P=.225)$, whereas a high $\mathrm{CCI}=3+$ score was associated with a significantly $16.4 \%$ higher likelihood of increased LoS compared with a CCI $=0$ score $(16.4 \%, 95 \%$ CI 0.079 to $0.256 ; P<.001)$.

Compared with the no comorbid condition, the percentage change in LoS was significant among IS patients with dementia (66.8\%, 95\% CI 0.370 to $1.030 ; P<.001)$, chronic pulmonary disease $(16.7 \%, 95 \%$ CI 0.061 to $0.284 ; P=.002)$, congestive heart failure $(13.6 \%, 95 \%$ CI 0.048 to $0.232 ; P=.002)$, and diabetes without end-organ damage $(8.8 \%, 95 \%$ CI 0.027 to $0.153 ; P=.004)$.

\section{Hospital Costs}

Table 2 also shows average hospitalization costs, with the mean costs in the CCI $=0$ group RMB39407 (SD \pm RMB53 678.6); $\mathrm{CCI}=1$ group $\mathrm{RMB} 32686(\mathrm{SD} \pm \mathrm{RMB} 48474.1) ; \mathrm{CCI}=2$

Table 2. Data for Hospitalizations, Analyzed According to the CCI Score $(N=5114)$

\begin{tabular}{|c|c|c|c|c|c|c|}
\hline & \multicolumn{2}{|c|}{ Average LoS (days) } & \multicolumn{2}{|c|}{ Average Hospital Cost (RMB) } & \multicolumn{2}{|c|}{ Death } \\
\hline & Mean (SD) & $P$ Value & Mean (SD) & $P$ Value & No. (\%) & $P$ Value \\
\hline $\mathrm{CCl}$ score & & $<.001$ & & $<.001$ & & $<.001$ \\
\hline 0 & $17(13.2)$ & & 39407 (53 678.6) & & $131(5.7)$ & \\
\hline 1 & $16(13.8)$ & & $32686(48474.1)$ & & 78 (4.9) & \\
\hline 2 & $18(13.2)$ & & 38714 (51 411.7) & & $67(9.5)$ & \\
\hline \multicolumn{7}{|l|}{ Individual CCI diseases (Ref = Without comorbidity) } \\
\hline Myocardial infarction (weight $=1$ ) & $20(26.4)$ & .631 & $66871(67585.0)$ & $<.001$ & $14(22.2)$ & $<.001$ \\
\hline Congestive heart failure (weight $=1$ ) & $20(20.5)$ & .003 & 43334 (55 864.8) & .025 & $76(13.2)$ & $<.001$ \\
\hline Peripheral vascular disease $($ weight $=1$ ) & $17(15.7)$ & .785 & 30228 (47 272.7) & $<.001$ & $51(4.6)$ & $<.001$ \\
\hline Dementia (weight = 1) & $28(35.5)$ & .082 & $48100(92767.8)$ & .525 & $19(12.4)$ & .076 \\
\hline Chronic pulmonary disease $($ weight $=1$ ) & $20(19.6)$ & $<.001$ & 38941 (64 630.4) & .073 & $45(9.6)$ & .009 \\
\hline Ulcer disease (weight = 1) & $18(13.5)$ & .865 & 43471 (52 385.3) & .025 & $4(8.2)$ & .175 \\
\hline Mild liver disease (weight $=1$ ) & $16(10.8)$ & .634 & 35491 (44 789.1) & .163 & $8(2.6)$ & .762 \\
\hline Diabetes without end-organ damage (weight $=1$ ) & $18(15.4)$ & .110 & $34663(54450.3)$ & .590 & $72(7.5)$ & .002 \\
\hline Hemiplegia (weight = 2) & $36(21.1)$ & .106 & 57205 (65 431.0) & .032 & $0(0.0)$ & .554 \\
\hline Moderate or severe renal disease $($ weight $=2$ ) & $19(17.7)$ & $<.001$ & $49081(72738.2)$ & .066 & $51(25.6)$ & .285 \\
\hline Diabetes with end-organ damage (weight $=2$ ) & $19(16.4)$ & .055 & 39260 (43 334.4) & .008 & $12(11.3)$ & .000 \\
\hline Tumor without metastasis (weight $=2$ ) & $19(11.2)$ & .858 & 60971 (59 852.1) & .207 & $32(21.3)$ & .083 \\
\hline Leukemia (weight = 2) & $8(3.5)$ & $<.001$ & 68841 (64 074.4) & $<.001$ & $2(100.0)$ & $<.001$ \\
\hline Lymphoma (weight = 2) & $46(51.3)$ & .088 & 102382 (104 227.9) & .240 & $1(16.7)$ & $<.001$ \\
\hline Moderate or severe liver disease $($ weight $=3$ ) & $20(18.2)$ & .062 & 67425 (75 159.7) & .005 & $10(18.9)$ & .358 \\
\hline Metastatic solid tumor (weight = 6) & $16(8.3)$ & .684 & 51599 (33 116.5) & $<.001$ & $14(34.1)$ & .001 \\
\hline AIDS (weight = 6) & $9(3.5)$ & .407 & $19230(6483.2)$ & $<.001$ & $0(0.0)$ & $<.001$ \\
\hline
\end{tabular}

Abbreviations: LoS, length of stay; $\mathrm{CCl}$, Charlson Comorbidity Index; SD, standard deviation; RMB, renminbi. 


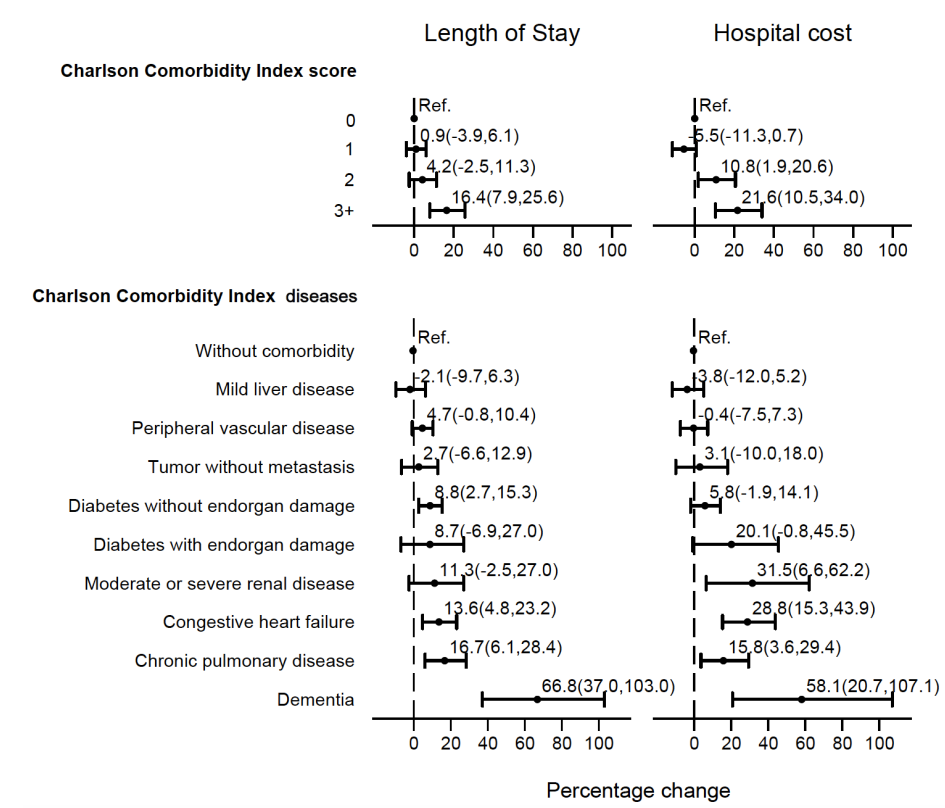

Figure 1. Percentage Change and $95 \% \mathrm{Cl}$ in LoS and Hospital Cost for IS Associated With Comorbid Conditions Adjusted for Gender, Age, Year of Diagnosis, Education Level, and Type of Insurance. Abbreviations: LoS, length of stay; SD, standard deviation; IS, ischemic stroke; Cl, confidence interval.

group RMB38714 (SD \pm RMB51 411.7); and CCI = 3+ group RMB48329 (SD \pm RMB71 150.2). Figure 1 presents the percentage change and $95 \% \mathrm{CI}$ in hospital cost associated with comorbid conditions. As shown in Table S1, after adjusting for control variables, the CCI $=1$ group $(-0.055,95 \%$ CI -0.113 to $0.007 ; P=.083)$ was not significant, but the $\mathrm{CCI}=2$ group $(0.108,95 \%$ CI 0.019 to $0.206 ; P=.017)$ was associated with a $10.8 \%$, and CCI $=3+$ group $(0.216,95 \%$ CI 0.105 to 0.340 ; $P<.001)$ a $21.6 \%$, higher likelihood of increased hospital costs compared with a $\mathrm{CCI}=0$ scores. Compared with no comorbidity, the percentage change was most notable among IS patients with dementia $(58.1 \%$, 95\% CI 0.207 to 1.071 ; $P<.001)$, moderate or severe renal disease $(31.5 \%, 95 \% \mathrm{CI}$ 0.066 to $0.622 ; P=.011)$, congestive heart failure $(28.8 \%, 95 \%$ CI 0.153 to $0.439 ; P<.001)$, and chronic pulmonary disease $(15.8 \%, 95 \%$ CI 0.036 to $0.294 ; P=.009)$.

\section{Mortality}

The overall death rate was $7.06 \%$, with the $\mathrm{CCI}=0$ group accounting for $5.7 \%$ of deaths, the $\mathrm{CCI}=1$ group accounting for $4.9 \%$ of deaths, CCI $=2$ group accounting for $9.5 \%$ of deaths and $\mathrm{CCI}=3+$ accounting for $16.4 \%$ of deaths. Figure 2 shows that the cumulative survival by CCI group, with patients with high comorbidity $(\mathrm{CCI}=3+)$ having significantly poorer survival rates than lower CCI groups. As shown in Table 3 , compared with $\mathrm{CCI}=0$ group, there was no significant difference between the $\mathrm{CCI}=1$ group (HR $0.789,95 \%$ CI 0.590 to $1.055 ; P=.110)$ and $\mathrm{CCI}=2$ group (HR $1.356,95 \%$ CI 0.989 to $1.859 ; P=.059)$ in the Cox regression model after adjusting for confounders. However, as shown in Table 3 , a high CCI $=3+$ score was positively correlated with approximately a 2.5 -fold higher risk of death compared to a $\mathrm{CCI}=0$ score group after adjusting for confounders (HR $2.419,95 \%$ CI 1.808 to $3.235 ; P<.001)$.

As shown in Table 3, the relative risk of death from all causes were most notable among IS with moderate or severe renal disease (HR 3.992, 95\% CI 2.795 to 5.702; $P<.001$ ), followed by patients with tumor without metastasis (HR 3.361, 95\% CI 2.214 to $5.101 ; P<.001$ ), congestive heart failure (HR 1.837, 95\% CI 1.341 to $2.517 ; P<.001)$, diabetes with end-organ damage (HR 1.986, 95\% CI 1.061 to $3.716 ; P=.032$ ), and peripheral vascular disease (HR $0.656,95 \%$ CI 0.466 to 0.925 ; $P=.016)$.

\section{Discussion}

Our study systematically examined the associations between comorbidity and LoS, hospital costs, and mortality among immobile IS patients. Overall, $55.2 \%$ of our sample's IS

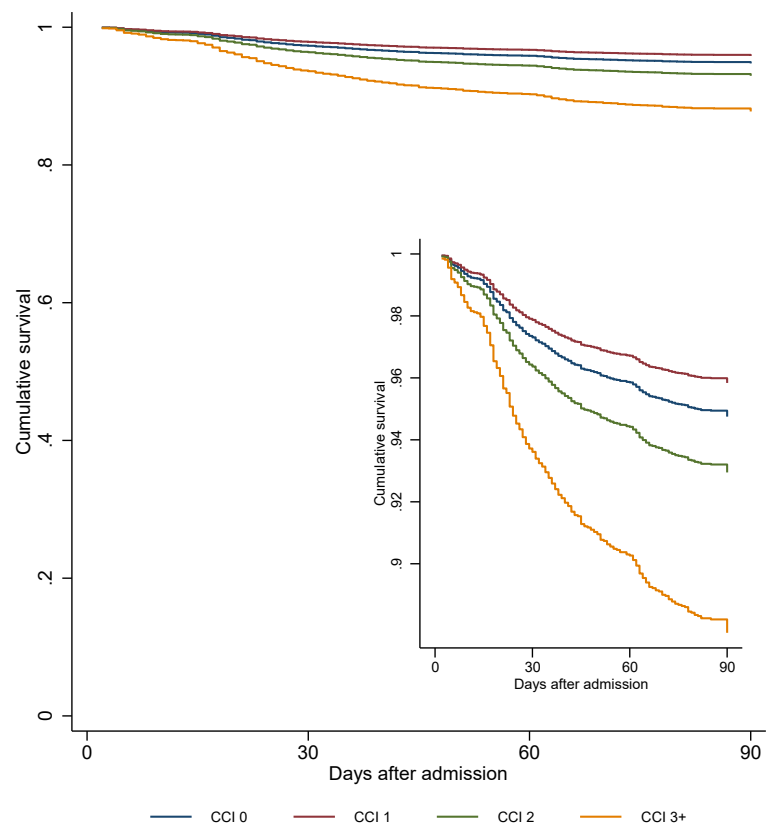

Figure 2. Cumulative Survival by $\mathrm{CCl}$ Score and Adjusted for Gender, Age, Education Level, and Type of Insurance. The smaller figure within Figure 2, adjusted the starting point of the vertical axis to present the differences between the groups more prominently. Abbreviation: $\mathrm{CCl}$, Charlson Comorbidity Index. 
Table 3. Results From Cox Regression Model

\begin{tabular}{ll}
\hline Variable & HR (95\% Cl) \\
\hline CCl score (Ref. = $)$ & $0.789(0.590-1.055)$ \\
1 & $1.356(0.989-1.859)$ \\
2 & $2.419(1.808-3.235)^{\mathrm{a}}$ \\
$3+$ & $0.656(0.466-0.925)^{\mathrm{a}}$ \\
Individual CCl diseases (Ref. = Without comorbidity) \\
Peripheral vascular disease & $1.282(0.947-1.734)$ \\
Diabetes without end-organ damage & $1.837(1.341-2.517)^{\mathrm{b}}$ \\
Congestive heart failure & $1.221(0.843-1.768)$ \\
Chronic pulmonary disease & $0.521(0.254-1.069)$ \\
Mild liver disease & $3.992(2.795-5.702)^{\mathrm{a}}$ \\
Moderate or severe renal disease & $1.488(0.871-2.543)$ \\
Dementia & $3.361(2.214-5.101)^{\mathrm{a}}$ \\
Tumor without metastasis & $1.986(1.061-3.716)^{\mathrm{b}}$ \\
\hline
\end{tabular}

Abbreviations: $\mathrm{CCl}$, Charlson Comorbidity Index; $\mathrm{HR}$, hazard ratio; $\mathrm{Cl}$, confidence interval. All models adjusted for gender, age, year of diagnosis, education level, and type of insurance.

${ }^{\mathrm{a}} P<.001,{ }^{\mathrm{b}} P<.05$.

patients had a comorbid condition, with a high CCI score associated with increased average LoS, higher hospital costs, and a greater likelihood of death.

The prevalence estimates of comorbidities among immobile IS patients in China was $55.2 \%$, which is lower than a retrospective analysis extracted from the Sichuan Provincial People's Hospital database reporting that $79 \%$ of inpatients with IS had at least one comorbidity. ${ }^{23}$ Previous research indicated that $94.2 \%$ of stroke sufferers have one or more comorbid conditions in Scotland, ${ }^{45}$ and the prevalence of comorbidity ranged from $44 \%$ in Denmark ${ }^{34}$ to $99 \%$ in Ontario, Canada. ${ }^{46}$ These differences are likely due to a variety of factors, including the study design, ${ }^{23}$ study periods,${ }^{34}$ study subjects, ${ }^{46}$ local factors (such as the standard of nursing care or medical treatment received), ${ }^{33}$ and the use of different classifications or ICD codes for determining comorbidities. ${ }^{16}$

We found the most frequent comorbid condition was peripheral vascular disease $(21 \%)$. There are a number of possible explanations for this situation. Participants enrolled in our study were immobile, and the literature indicates that peripheral vascular disease are closely related to immobilization. ${ }^{14,47}$ Second, there are some common potential risk factors for both peripheral vascular disease and IS, including hypertension, alcohol assumption and atherosclerosis. ${ }^{48-51}$ We cannot, however, infer the sequence and the causality of IS and peripheral vascular disease. Since vascular examinations were routinely conducted for immobile patients (especially for those with suspected cardiovascular and cerebrovascular diseases), tests such as Doppler ultrasound, computed tomography angiography, magnetic resonance angiography, may contribute to the higher prevalence of peripheral vascular disease in our study. ${ }^{23}$ This result suggests that early tests, such as Doppler ultrasound, may improve the detection rate of peripheral vascular disease in immobile IS patients, and prospective clinical study is required to justify this issue.
Our results suggested that a $\mathrm{CCI}=2$ score was related to higher hospital costs, and a high $\mathrm{CCI}=3+$ score was positively correlated with both longer LoS and higher hospital costs, which supports CCI as a measure for the disease burden of IS. ${ }^{26,33}$ Since CCI $=3+$ group had a higher likelihood of increased LoS and hospital costs compared with a CCI $=0$ score, with similar results for the $\mathrm{CCI}=1$ group and $\mathrm{CCI}=2$ group, $\mathrm{CCI}=3+$ patients need more attention during hospitalization. Dementia, chronic pulmonary disease, and congestive heart failure were LoS and hospital cost-specific comorbidities. One explanation is that approximately three in four participants enrolled in our study were the elderly, and these chronic diseases tend to cluster in cohorts with increasing age.

We also found that high comorbidity as measured by the $\mathrm{CCI}=3+$ was associated with an approximately 2.5 -fold higher risk of death than a low $\mathrm{CCI}=0$ score. This confirms the use of CCI to predict short-term death in the Chinese IS population is meaningful. Our results also indicate that moderate or severe renal disease and tumor without metastasis increased the death rate. This can be explained by stroke rates for end-stage renal disease patients and cancer are substantially higher than for the general population, and those renal and cancer patients have a poor prognosis and high mortality rate, despite advances in diagnosis and treatment. ${ }^{15,52}$

The association between the scores of CCI and multiple clinical outcomes, including LoS, hospital costs, and mortality, supports the notion that the CCI should be used to identify and manage chronic comorbidities, ${ }^{16,22,33,36}$ which identify patients with multiple chronic diseases in a universally applicable, transparent, and auditable method, through a weighted measure of the burden of chronic disease that predicts long term prognosis, outcomes and costs. ${ }^{53,54}$ In addition, the CCI was designed to enable researchers to control for the prognostic impact of other chronic diseases on the health outcomes of patients with a specific chronic disease. Without CCI, researchers often excluded patients with other chronic diseases from their studies to eliminate the potential of 'confounding' factors. For instance, studies of patients with diabetes might exclude patients with dementia or cancer to make certain that they did not confound the clinical outcomes. Such exclusions limited the number of participants to whom the study results applied. ${ }^{31,34}$ Further, reference to a CCI indicator is both a simple and more direct method than a nonstandard list of a variety of different chronic disease conditions, which facilitates doctors' decisionmaking. Therefore, knowing a patient has a CCI score of 3+ provides more useful information than just knowing if the patient has hypertension or dementia. In the future, health insurance plans should require and use the CCI to identify the complexity of stroke-related diseases and the current chronic disease status (such as hypertension, dementia and diabetes) of the insured member. Based on the stratification of zero, one, two, and three or more CCI diseases, and the patients' comorbid conditions, the government should increase the financial investment, raise reimbursement rates and set up differential reimbursements to meet the health needs of patients with multiple diseases. For clinical staff, CCI should 
be used to assess, monitor, and treat comorbidities during hospitalization..$^{55}$ The early use of the CCI plays a critical role in the identification of IS patients most likely to experience the worse clinical outcomes and may help target interventions according to the resulting risk information, which can tackle such conditions and reduce their pernicious effects.

China is encountering formidable healthcare challenges related to population aging, with $12 \%$ of the population over 65 years old in 2020, which is forecast to rise to $26 \%$ by $2050 .{ }^{56}$ Comorbidities associated with the aging population will pose a significant challenge to China's health system. Combined with tailored stroke interventions according to patient comorbidity, we recommend the establishment of a geriatric care system to improve the health of the strokeprone population and reduce the stroke burden on the health system.

Our study has some limitations. Common to health research, a retrospective post-hoc analysis study design was used in the current study, where existing data was analyzed to answer new questions. For example, some clinical data, such as the details of the cause of death, laboratory data, clinical severity of stroke and premorbid Rankin scale data were not recorded in the HIS, which is an inherent drawback in retrospective analysis of a prospectively collected database. Second, no data on the severity of comorbidities was available. Third, we cannot infer the sequence and the causality of IS and peripheral vascular disease. Fourth, diagnostic validity of comorbidity might be influenced by heterogeneity of physician documentation and code assignment accuracy from different sites. Prospective studies with more sophisticated evaluations are required to address these limitations.

\section{Conclusion}

Comorbidities were central drivers of LoS, hospital cost, and mortality in Chinese immobile IS patients. We found CCI had a significant predictive value for clinical and economic outcomes in IS patients. Due to population aging, the CCI should be used to identify, monitor and manage chronic comorbidities among the immobile IS population. We also recommend establishing a geriatric care system, where the CCI can be optimized in patient treatment.

\section{Acknowledgements}

The authors thank research participants and nursing staff for their kind and efficient contribution to the study. We acknowledge the helpful comments by three anonymous reviewers.

\section{Ethical issues}

The Ethics Committee of Peking Union Medical College Hospital (S-700) approved the project. All patients provided written informed consent before enrollment in the study, and all IS patients coded I63.x according to the ICD-10 enrolled in the current study $(N=5114)$. Patients' records and information were anonymized and de-identified prior to the analysis.

\section{Competing interests}

Authors declare that they have no competing interests.

\section{Authors' contributions}

Study concept and design: XW. Analysis and interpretation of data: HL and DZ. Editing of the manuscript and drafting of tables: HL and DZ. Critical review of the manuscript for important intellectual content: XW, HL, and DZ. Patient recruitment, data collection, and manuscript editing: BS, JJ, YL, XW, SC, EM and $\mathrm{SN}$. All authors critically reviewed and approved the manuscript before it was submitted.

\section{Authors' affiliations}

${ }^{1}$ Department of Nursing, Chinese Academy of Medical Sciences - Peking Union Medical College, Peking Union Medical College Hospital, Beijing, China. 'Department of Nursing, Henan Provincial People's Hospital, Zhengzhou, China. ${ }^{3}$ Department of Nursing, The Second Affiliated Hospital Zhejiang University School of Medicine, Hangzhou, China. ${ }^{4}$ Department of Nursing, Wuhan Union Hospital, Wuhan, China. ${ }^{5}$ Department of Nursing, Sichuan Provincial People's Hospital, Chengdu, China. ${ }^{6}$ Department of Nursing, The First Affiliated Hospital, Sun Yat-sen University, Guangzhou, China. ${ }^{7}$ Australian National Institute of Management and Commerce, Sydney, NSW, Australia. ${ }^{8}$ School of Economics and School of Management, Tianjin Normal University, Tianjin, China. ${ }^{9}$ Guangdong Institute for International Strategies, Guangdong University of Foreign Studies, Guangzhou, China. ${ }^{10}$ Newcastle Business School, University of Newcastle, Newcastle, NSW, Australia. ${ }^{11}$ School of Management, University of Liverpool, Liverpool, UK. ${ }^{12}$ China Center for Health Development Studies, Peking University, Beijing, China.

\section{Supplementary files}

Supplementary file 1 contains Table S1.

\section{References}

1. Scrutinio D, Ricciardi C, Donisi L, et al. Machine learning to predict mortality after rehabilitation among patients with severe stroke. Sci Rep. 2020;10(1):20127. doi:10.1038/s41598-020-77243-3

2. Hankey GJ. Stroke. Lancet. 2017;389(10069):641-654. doi:10.1016/ s0140-6736(16)30962-x

3. Iuppariello L, D'Addio G, Romano M, et al. Efficacy of the Regent Suitbased rehabilitation on gait EMG patterns in hemiparetic subjects: a pilot study. Eur J Phys Rehabil Med. 2018;54(5):705-716. doi:10.23736/s19739087.18.04706-8

4. Johnson CO, Nguyen M, Roth GA, et al. Global, regional, and national burden of stroke, 1990-2016: a systematic analysis for the Global Burden of Disease Study 2016. Lancet Neurol. 2019;18(5):439-458. doi:10.1016/ s1474-4422(19)30034-1

5. Wang $\mathrm{W}$, Jiang $\mathrm{B}$, Sun $\mathrm{H}$, et al. Prevalence, incidence, and mortality of stroke in China: results from a nationwide population-based survey of 480687 adults. Circulation. 2017;135(8):759-771. doi:10.1161/ circulationaha.116.025250

6. Li Z, Jiang Y, Li H, Xian Y, Wang Y. China's response to the rising stroke burden. BMJ. 2019;364:I879. doi:10.1136/bmj.1879

7. Wu S, Wu B, Liu M, et al. Stroke in China: advances and challenges in epidemiology, prevention, and management. Lancet Neurol. 2019; 18(4):394-405. doi:10.1016/s1474-4422(18)30500-3

8. Ekker MS, Boot EM, Singhal AB, et al. Epidemiology, aetiology, and management of ischaemic stroke in young adults. Lancet Neurol. 2018; 17(9):790-801. doi:10.1016/s1474-4422(18)30233-3

9. Wu X, Zou S, Zhu B, Shi J. The hospital costs of stroke patients in Chinese island populations: an 11-year tendency analysis. J Stroke Cerebrovasc Dis. 2015;24(5):988-992. doi:10.1016/j.jstrokecerebrovasdis.2014.12.021

10. Yong $M$, Xianjun $\mathrm{X}$, Jinghu L, Yunyun F. Effect of health insurance on direct hospitalisation costs for in-patients with ischaemic stroke in China. Aust Health Rev. 2018;42(1):39-44. doi:10.1071/ah16056

11. Tian $\mathrm{Y}$, Liu $\mathrm{H}$, Zhao Z, et al. Association between ambient air pollution and daily hospital admissions for ischemic stroke: a nationwide timeseries analysis. PLoS Med. 2018;15(10):e1002668. doi:10.1371/journal. pmed. 1002668

12. Corraini P, Ording AG, Henderson VW, Szépligeti S, Horváth-Puhó E, Sørensen HT. Cancer, other comorbidity, and risk of venous thromboembolism after stroke: a population-based cohort study. Thromb Res. 2016;147:88-93. doi:10.1016/j.thromres.2016.09.029

13. Aarnio K, Haapaniemi E, Melkas S, Kaste M, Tatlisumak T, Putaala J. Long-term mortality after first-ever and recurrent stroke in young adults. Stroke. 2014;45(9):2670-2676. doi:10.1161/strokeaha.114.005648

14. Kumar S, Selim MH, Caplan LR. Medical complications after stroke. Lancet Neurol. 2010;9(1):105-118. doi:10.1016/s1474-4422(09)70266-2

15. Corraini $P$, Szépligeti SK, Henderson VW, Ording AG, HorváthPuhó E, Sørensen HT. Comorbidity and the increased mortality after 
hospitalization for stroke: a population-based cohort study. J Thromb Haemost. 2018;16(2):242-252. doi:10.1111/jth.13908

16. Bar B, Hemphill JC 3rd. Charlson comorbidity index adjustment in intracerebral hemorrhage. Stroke. 2011;42(10):2944-2946. doi:10.1161/ strokeaha.111.617639

17. Liu L, Wang D, Wong KS, Wang Y. Stroke and stroke care in China: huge burden, significant workload, and a national priority. Stroke. 2011; 42(12):3651-3654. doi:10.1161/strokeaha.111.635755

18. Egerton T, Hokstad A, Askim T, Bernhardt J, Indredavik B. Prevalence of fatigue in patients 3 months after stroke and association with early motor activity: a prospective study comparing stroke patients with a matched general population cohort. BMC Neurol. 2015;15:181. doi:10.1186/ s12883-015-0438-6

19. Liu H, Zhu D, Cao J, et al. The effects of a standardized nursing intervention model on immobile patients with stroke: a multicenter study in China. Eur J Cardiovasc Nurs. 2019;18(8):753-763. doi:10.1177/1474515119872850

20. Widdicombe JG, Addington WR, Fontana GA, Stephens RE. Voluntary and reflex cough and the expiration reflex; implications for aspiration after stroke. Pulm Pharmacol Ther. 2011;24(3):312-317. doi:10.1016/j. pupt.2011.01.015

21. Liu H, Zhu D, Song B, et al. Cost-effectiveness of an intervention to improve the quality of nursing care among immobile patients with stroke in China: a multicenter study. Int J Nurs Stud. 2020;110:103703. doi:10.1016/j.ijnurstu.2020.103703

22. Lin CY, Chien CC, Chen HA, et al. The impact of comorbidity on survival after hemorrhagic stroke among dialysis patients: a nationwide populationbased study. BMC Nephrol. 2014;15:186. doi:10.1186/1471-2369-15-186

23. Si $Y$, Xiao X, Xiang $S$, et al. Mortality-specific comorbidity among inpatients with ischemic stroke in West China. Acta Neurol Scand. 2019;140(2):100106. doi:10.1111/ane.13108

24. Zhao Y, Condon J, Lawton P, He V, Cadilhac DA. Lifetime direct costs of stroke for indigenous patients adjusted for comorbidities. Neurology. 2016;87(5):458-465. doi:10.1212/wnl.0000000000002908

25. Rutten-Jacobs LC, Arntz RM, Maaijwee NA, et al. Long-term mortality after stroke among adults aged 18 to 50 years. JAMA. 2013;309(11):11361144. doi:10.1001/jama.2013.842

26. Lim JH, Cheon $\mathrm{SH}$. Analysis of variation in length of stay (LOS) after ischemic and hemorrhagic stroke using the Charlson Comorbidity Index (CCI). J Phys Ther Sci. 2015;27(3):799-803. doi:10.1589/jpts.27.799

27. Kokotailo RA, Hill MD. Coding of stroke and stroke risk factors using international classification of diseases, revisions 9 and 10. Stroke. 2005; 36(8):1776-1781. doi:10.1161/01.STR.0000174293.17959.a1

28. Ricciardi C, Balato G, Romano M, Santalucia I, Cesarelli M, Improta G. Fast track surgery for knee replacement surgery: a lean six sigma approach. TQM J. 2020;32(3):461-474. doi:10.1108/TQM-06-2019-0159

29. Improta G, Balato G, Romano M, et al. Lean Six Sigma: a new approach to the management of patients undergoing prosthetic hip replacement surgery. J Eval Clin Pract. 2015;21(4):662-672. doi:10.1111/jep.12361

30. Latessa I, Ricciardi C, Jacob D, et al. Health technology assessment through Six Sigma Methodology to assess cemented and uncemented protheses in total hip arthroplasty. Eur J Transl Myol. 2021;31(1):9651. doi:10.4081/ejtm.2021.9651

31. Charlson ME, Pompei P, Ales KL, MacKenzie CR. A new method of classifying prognostic comorbidity in longitudinal studies: development and validation. J Chronic Dis. 1987;40(5):373-383. doi:10.1016/00219681(87)90171-8

32. Liu H, Wu X, Cao J, et al. Effect of comorbidity assessed by the Charlson comorbidity index on the length of stay and mortality among immobile hemorrhagic stroke patients younger than 50 years. Front Neurol. 2020; 11:487. doi: $10.3389 /$ fneur.2020.00487

33. Ofori-Asenso R, Zomer E, Chin KL, et al. Effect of comorbidity assessed by the Charlson comorbidity index on the length of stay, costs and mortality among older adults hospitalised for acute stroke. Int J Environ Res Public Health. 2018;15(11):2532. doi:10.3390/ijerph15112532

34. Schmidt M, Jacobsen JB, Johnsen SP, Bøtker HE, Sørensen HT. Eighteenyear trends in stroke mortality and the prognostic influence of comorbidity. Neurology. 2014;82(4):340-350. doi:10.1212/wnl.0000000000000062

35. Jacobs DR Jr, Kroenke C, Crow R, et al. PREDICT: a simple risk score for clinical severity and long-term prognosis after hospitalization for acute myocardial infarction or unstable angina: the Minnesota heart survey. Circulation. 1999;100(6):599-607. doi:10.1161/01.cir.100.6.599

36. Falsetti L, Viticchi G, Tarquinio N, et al. Charlson comorbidity index as a predictor of in-hospital death in acute ischemic stroke among very old patients: a single-cohort perspective study. Neurol Sci. 2016;37(9):14431448. doi:10.1007/s10072-016-2602-1

37. Jiménez Caballero PE, López Espuela F, Portilla Cuenca JC, Ramírez Moreno JM, Pedrera Zamorano JD, Casado Naranjo I. Charlson comorbidity index in ischemic stroke and intracerebral hemorrhage as predictor of mortality and functional outcome after 6 months. J Stroke Cerebrovasc Dis. 2013;22(7):e214-218. doi:10.1016/j. jstrokecerebrovasdis.2012.11.014

38. Fang $\mathrm{H}$, Eggleston $\mathrm{K}$, Hanson $\mathrm{K}, \mathrm{Wu} \mathrm{M}$. Enhancing financial protection under China's social health insurance to achieve universal health coverage. BMJ. 2019;365:I2378. doi:10.1136/bmj.I2378

39. Chen J, Yu H, Dong H. Effect of the new rural cooperative medical system on farmers' medical service needs and utilization in Ningbo, China. BMC Health Serv Res. 2016;16(1):593. doi:10.1186/s12913-016-1842-3

40. Lin W, Liu GG, Chen G. The Urban Resident Basic Medical Insurance: a landmark reform towards universal coverage in China. Health Econ. 2009;18 Suppl 2:S83-96. doi:10.1002/hec.1500

41. Zhou Z, Zhu L, Zhou Z, Li Z, Gao J, Chen G. The effects of China's urban basic medical insurance schemes on the equity of health service utilisation: evidence from Shaanxi Province. Int J Equity Health. 2014 13:23. doi:10.1186/1475-9276-13-23

42. Zhang X, Qiu H, Liu S, Li J, Zhou M. Prediction of prolonged length of stay for stroke patients on admission for inpatient rehabilitation based on the international classification of functioning, disability, and health (ICF) generic set: a study from 50 centers in China. Med Sci Monit. 2020; 26:e918811. doi:10.12659/msm.918811

43. Yin X, Huang L, Man X, et al. Inpatient cost of stroke in Beijing: a descriptive analysis. Neuroepidemiology. 2018;51(3-4):115-122. doi:10.1159/000491091

44. Yang Y, Nicholas S, Li S, et al. Health care utilization for patients with stroke: a 3-year cross-sectional study of China's two urban health insurance schemes across four cities. BMC Public Health. 2021;21(1):531. doi:10.1186/s12889-021-10456-x

45. Gallacher KI, Batty GD, McLean G, et al. Stroke, multimorbidity and polypharmacy in a nationally representative sample of 1,424,378 patients in Scotland: implications for treatment burden. BMC Med. 2014:12:151. doi:10.1186/s12916-014-0151-0

46. Gruneir A, Griffith LE, Fisher K, et al. Increasing comorbidity and health services utilization in older adults with prior stroke. Neurology. 2016; 87(20):2091-2098. doi:10.1212/wnl.0000000000003329

47. Balkanay $\mathrm{OO}$, Ömeroğlu SN. [Approach to peripheral arterial disease in the elderly]. Turk Kardiyol Dern Ars. 2017;45(Suppl 5):96-101. doi:10.5543/tkda.2017.08444

48. Makin A, Lip GY, Silverman S, Beevers DG. Peripheral vascular disease and hypertension: a forgotten association? J Hum Hypertens. 2001; 15(7):447-454. doi:10.1038/sj.jhh.1001209

49. Moerch-Rasmussen A, Nacu A, Waje-Andreassen U, Thomassen L, Naess $\mathrm{H}$. Recurrent ischemic stroke is associated with the burden of risk factors. Acta Neurol Scand. 2016;133(4):289-294. doi:10.1111/ane.12457

50. Diener HC, Hankey GJ. Primary and secondary prevention of ischemic stroke and cerebral hemorrhage: JACC focus seminar. J Am Coll Cardiol. 2020;75(15):1804-1818. doi:10.1016/j.jacc.2019.12.072

51. Wakabayashi I, Sotoda Y. [Alcohol drinking and peripheral arterial disease of lower extremity]. Nihon Arukoru Yakubutsu Igakkai Zasshi. 2014;49(1):13-27.

52. Seliger SL, Gillen DL, Longstreth WT Jr, Kestenbaum B, Stehman-Breen $\mathrm{CO}$. Elevated risk of stroke among patients with end-stage renal disease. Kidney Int. 2003;64(2):603-609. doi:10.1046/j.1523-1755.2003.00101.x

53. Charlson M, Szatrowski TP, Peterson J, Gold J. Validation of a combined comorbidity index. J Clin Epidemiol. 1994;47(11):1245-1251. doi:10.1016/0895-4356(94)90129-5

54. Pompei P, Charlson ME, Douglas RG Jr. Clinical assessments as predictors of one year survival after hospitalization: implications for prognostic stratification. J Clin Epidemiol. 1988;41(3):275-284 . doi:10.1016/0895-4356(88)90132-1

55. Kurth T, Berger K. The socioeconomic stroke puzzle. Stroke. 2007;38(1):4 5. doi:10.1161/01.STR.0000252874.11524.cc

56. Yang G, Kong L, Zhao W, et al. Emergence of chronic non-communicable diseases in China. Lancet. 2008;372(9650):1697-1705. doi:10.1016/ s0140-6736(08)61366-5 